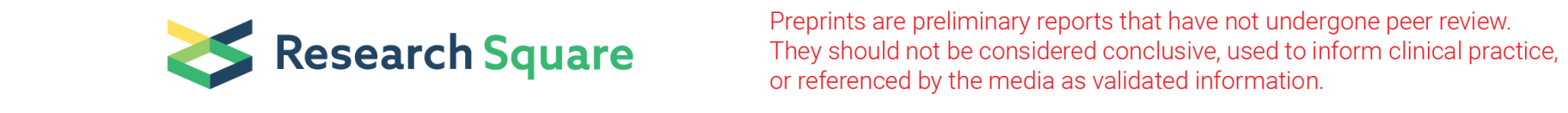

\title{
Solitary Drinking in South Korea: Are the Drinking Norms, Motives, and Alcohol Consumption of Solitary Drinkers Different from those of Social Drinkers?
}

\author{
Jumoon Park \\ Incheon National University \\ Sohn Aeree ( $\nabla$ aeree@syu.ac.kr) \\ Sahmyook University https://orcid.org/0000-0002-8524-2433
}

Research

Keywords: solitary drinking, social drinking, norms, motive

Posted Date: April 2nd, 2020

DOl: https://doi.org/10.21203/rs.3.rs-18791/v1

License: (9) This work is licensed under a Creative Commons Attribution 4.0 International License. Read Full License 


\section{Abstract}

Background: This study aimed to clarify the differences in drinking norms and motives among different groups (non/light, solitary, and social drinkers) in a representative sample of Korean adults. We also examined which group-solitary or social drinkers-was more likely to engage in heavy drinking.

Methods: The study used nationwide cross-sectional data collected in 2018. A total of 3,000 subjects aged 19 to 60 years were sampled for this study, including 1,532 men and 1,469 women. The data were analyzed with SPSS 23.0.

Results: Solitary drinkers are more likely to be divorced or separated, less educated, and marginally employed (people with temporary jobs, rather than regular; the self-employed; and people with service- and sales-related jobs). Solitary drinkers peaked in their $30 \mathrm{~s}(18.5 \%)$ and social drinkers in their $50 \mathrm{~s}$ (68.1\%). Solitary drinkers drank more frequently than social drinkers (Mean=6.1 vs. 3.6 per month, $\mathrm{p}<.001$ ) and consumed a significantly larger quantity of alcohol (Mean=69.5 g vs. $46.8 \mathrm{~g}$ per week). S olitary drinkers were more accepting of drinking-related behaviors in a variety of situations than were social drinkers. The regression analysis revealed that personal motives for drinking were the most important factor of drinking frequency and drinking consumption for both solitary and social drinking.

Conclusion: These findings suggest that people who drink alone may be more vulnerable to alcohol abuse than social drinkers.

\section{Background}

Alcohol consumption worsens many health conditions, from injuries to chronic diseases, and causes third-party harm through violence and accidents. Globally, alcohol is the third largest risk factor for disease and disability, contributing to the number of fatal injuries as well as $5.38 \%$ of all deaths and $5.1 \%$ of all disability-adjusted life years lost. Alcohol consumption also leads to many social problems, including violent crimes, accidents and injuries, and unwanted behaviors [1-2].

In 2015, the reported annual per capita alcohol consumption of people aged 15 years and over in Korea was 9.1 liters, which is slightly higher than the OECD mean of 9.0 liters [3] and world mean of 6.4 liters 157[1]. Alcohol consumption in Korea accounts for a significant proportion of disease and injury. In 2011, the net total of 4,393 deaths attributed nationally to alcohol consumption accounted for $1.9 \%$ of all deaths. The socioeconomic cost of alcohol consumption reached approximately 7.3698 trillion won in 2012 [4].

Among Korean adults aged 19 years and older, $59.2 \%$ (73.1\% of men and $45.4 \%$ of women) consumed alcohol monthly in 2017 [5]. About $50.8 \%$ of men and $26.9 \%$ of women reported binge drinking, defined as consuming seven (five) or more on one drinking occasion for men (women) in the past month 169[5]. A standard drink in South Korea contains 7-8 grams of pure alcohol. In Korea, $20 \%$ of drinkers are binge or excessive drinkers responsible for $66 \%$ of all alcohol consumption [6]. Harmful use of alcohol through excessive or binge drinking has been identified by the Institute for Health Metrics and Evaluation as the fifth largest risk factor for death and disability combined. However, the Korean government has invested little effort in preventing and reducing alcohol consumption [7].

A society's beliefs about alcohol and drinking behaviors are part of its social norms [8]. Behaviors related to drinking reflect the history, culture, religion, lifestyle, and racial characteristics of each ethnic group or nationality [9]. An individual's attitude and behaviors pertaining to drinking constitute his or her social behavior, irrespective of the style of drinking (solitary or social). Drinking motives are more important to drinking behavior than alcohol consumption expectation that influence choices, patterns, and outcomes related to alcohol [10]. Drinking motives are considered to play a role in drinking contexts. The two reasons for alcohol use according to motivational models were: 1) to enhance positive emotions and 2) to reduce negative emotions, which represent psychologically distinct and strategically motivated behaviors. Solitary drinkers use alcohol to cope with negative affect, while social drinkers use it to enhance positive affects [11-13]. However, these same authors also found that solitary drinkers were higher in all of positive or negative expectancies [12-13]. 
The most important of such cross-cultural constants in social norms governing alcohol use is the near-universal taboo on solitary drinking. In almost all cultures, drinking is essentially a social act. It is the same in Korea, where drinking has traditionally been a social behavior [14]. Accordingly, drinking alone has been seen as an indication of the drinker developing a problem that is abnormal or symptomatic of depression or interpersonal challenges [15]. There are few cultures in which drinking alone is actively approved or encouraged. The use of alcohol for a reason (drinking motive) and drinking patterns may help explain whether solitary drinking is a problem.

Recently, the popularity of solitary drinking has grown in Korea [15] owing to increasing numbers of people living alone. According to the Korean Food and Drug Administration (KFDA), two-thirds of all Korean adults reported having consumed alcohol alone within the past six months [16]. Solitary drinking is not in itself a problematic behavior. However, when solitary drinkers consume large quantities of alcohol, their risk increases. In Korea, few studies have been conducted on solitary drinkers' socio-demographic factors, motives, and alcohol consumption, meaning that little is currently known about solitary drinkers. Few studies have examined the relationship between drinking motives and alcohol consumption in the context of both solitary alcohol use and social alcohol use in South Korea [7]. We therefore examined differences in socio-demographic status, drinking norms, motives, and alcohol consumption according to drinking type in a representative sample of Korean adults.

\section{Methods}

\section{Participants and Procedure}

To determine the sample size for this study, demographically proportioned stratified sampling (gender, age, and region) was carried out in April 2018, using a panel of participants enrolled in a Korean research company. The assigned number of samples was 3,300 , stratified by gender, age, and region to obtain a nationally representative sample. An invitation to the online survey titled the "National Korean Drinking Culture Study" was sent to 7,411 adults aged 19 to 60 years, randomly selected from the electoral roll. A total of 3,015 participants aged 19 to 60 years was sampled for this study, including 1,532 men and 1,469 women. The mean age of the final sample was 40.64 years $(S D=11.06)$. Approximately $55.5 \%$ of the participants were married. Prior to conducting the survey with a pre-prepared questionnaire, the protocol of this study was approved by our Institutional Review Board (2-104078-AB-N-01-2017105HR). Informed consent was obtained from all participants.

\section{Measures}

\section{Amount of alcohol consumption}

To measure participants' alcohol intake during the past month, questions on beverage-specific quantity frequency were identified. Participants indicated their typical monthly frequency of alcohol use and quantity (number drinks/occasion) over the past month. First, participants were asked about their number of drinking occasions during the past month. If they answered that they drank once or more per month, we asked about their preferred Korean alcoholic beverage (soju, beer, makgeolli, wine, or spirits) and then the quantity of alcohol on each occasion for the preferred types of alcoholic beverage (number drinks/occasion). For example, if the subject drank $500 \mathrm{cc}$ of beer, the calculation would be $500 \times 0.045$ (alcohol content $\%$ by volume) $\times 0.789$ (density) $=17.75$ [7]. This person would have consumed 17.75 grams of pure alcohol (ethanol), which represents his or her drinking quantity

\section{Alcohol-related harm}

We asked seven questions on alcohol-related harms or troubles in the past year (e.g., having trouble with work, blacking out) with possible responses of "No," "Once or twice," and "Three times or more" to each. We categorized the "No" responses as 0 and the "Yes" responses (one or more times) as 1.

\section{Type of drinker: Non-drinker, solitary drinker, or social drinker}


Non/light drinkers are those who do not drink or drink less than one drink per month. At the time of this study, there were few published measures of solitary drinking behavior. Therefore, we modified a single item developed by Christiansen et al. [12] and Gonzalez et al. [11]. The participants were asked to classify themselves as solitary or social drinkers. This question involved dichotomizing participants and simply reflected whether the respondent usually drank alone or with other people. Of course, a solitary drinker may also be a social drinker; however, for the study's purposes, he or she was classified exclusively as a solitary or social drinker.

\section{Social norms}

The drinking social norms questionnaire developed by Sohn [17] is comprised of seven self-report items such as, for example, whether respondents considered it acceptable to drink at a park or on a mountain, during the day, when under-aged, alone, and so on. The responses were coded using a five-point Likert-type scale, and the scores were summed to yield the total score; the higher the score, the more acceptable certain drinking behaviors were. Cronbach's coefficient (a) was 0.70.

\section{Drinking motives}

If participants answered that they drank once or more per month, we asked about their motives for drinking. The drinking motives questionnaire developed by Sohn [17] is comprised of 12 self-report items, measuring three dimensions of subscale domains. Personal drinking motives (six items) relate to enhancement of positive emotions (three items) and coping with negative affect (three items). In Sohn's study, enhancement of positive emotions and coping with negative motives were not different each other, so she bounded the same scale [17]. Social drinking motives (three items) relate to the improvement of sociability and interaction with others. Environmental drinking motives (three items) relate to stimulation by an environment containing many liquor stores and places to drink. Participants were asked to rate how often each statement reflected their reasons for drinking over the last 12 months, on a five-point Likert-type scale ranging from 1 (strongly disagree) to 5 (strongly agree). The drinking motives subscales demonstrated good convergent and concurrent validity [17]. Higher scores indicated strength of agreement with personal, social, and environmental drinking motives. Cronbach's coefficients for the personal, social, and environmental drinking motives scales were $0.84,0.83$, and 0.88 , respectively, showing relatively high reliability. We expected responses to each question to show significant differences between solitary and social drinkers

\section{Data analysis}

We utilized SPSS 24.0 (SPSS Inc., Chicago, IL, USA) for statistical analysis. The characteristics of different groups were analyzed using descriptive statistics (frequency, percentage, means, and standard deviation). The $\mathrm{X}_{\mathrm{MH}}{ }^{2}$ (Mantel-Haenszel) test with gender as a covariate for frequency and proportion was performed to compare sociodemographic characteristics for different groups. A multivariate analysis of covariance (MANCOVA), with gender as a covariate, was used to compare social and solitary drinkers in terms of frequency of drinking occasions per month and amount of alcohol consumption per week. Frequency of drinking occasions per month and amount of alcohol consumption were square-root-transformed to improve normality and reduce the influence of outliers.

\section{Results}

\section{Demographic characteristics of participants by drinking type}

Table 1. Demographic characteristics of participants by drinking type 




Note: ${ }^{*} \mathrm{p}<.05,{ }^{* *} \mathrm{p}<.01,{ }^{* * *} \mathrm{p}<.001$

\section{Social Norms By Drinking Type}

Table 2 shows that social norms regarding drinking differ by drinking type. Social norms for drinking shape common attitudes, perceptions, and behaviors. Except for two statements, solitary drinkers agreed with the statements more often than social drinkers. 
Table 2

Social drinking norms by drinking type (\% of agreement) $(\mathrm{N}=3,015)$

\begin{tabular}{|c|c|c|c|c|c|}
\hline & $\begin{array}{l}\text { Non/light } \\
(n=589) \\
\%\end{array}$ & $\begin{array}{l}\text { Solitary } \\
(n= \\
486) \\
\%\end{array}$ & $\begin{array}{l}\text { Social } \\
(n= \\
1,940) \\
\%\end{array}$ & $\begin{array}{l}\text { Total } \\
(\mathrm{N}= \\
3,015) \\
\%\end{array}$ & $\mathrm{X}_{\mathrm{MH}}^{2}$ \\
\hline $\begin{array}{l}\text { It is acceptable to drink in a park or on a mountain after } \\
\text { hiking. }\end{array}$ & 8.1 & 12.3 & 10.4 & 10.3 & $23.3^{\star \star \star}$ \\
\hline It is acceptable to drink during the day. & 25.1 & 37.0 & 31.5 & 31.1 & $63.9^{\star \star \star}$ \\
\hline It is acceptable to get drunk. & 25.0 & 42.6 & 37.9 & 36.2 & $85.4^{\star \star \star}$ \\
\hline It is acceptable for high school students to drink. & 3.7 & 4.1 & 4.4 & 4.2 & $13.1^{*}$ \\
\hline It is acceptable to drink alone. & 61.1 & 85.2 & 69.1 & 70.1 & $91.2^{\star \star \star}$ \\
\hline $\begin{array}{l}\text { Wrongdoing (or crimes) committed while drunk can be } \\
\text { forgiven. }\end{array}$ & 0.8 & 1.6 & 1.6 & 1.5 & 7.7 \\
\hline It is discourteous to refuse a drink offered by someone else. & 5.8 & 7.4 & 7.4 & 7.1 & 7.6 \\
\hline It is acceptable to drink in a convenience store. & 24.6 & 38.1 & 35.2 & 33.6 & $52.5^{\star \star \star}$ \\
\hline Mean (SD) & $\begin{array}{l}2.33 \\
(0.61)\end{array}$ & $\begin{array}{l}2.67 \\
(0.49)\end{array}$ & $\begin{array}{l}2.56 \\
(0.51)\end{array}$ & $\begin{array}{l}2.53 \\
(0.54)\end{array}$ & $\begin{array}{l}\mathrm{F}= \\
61.0^{\star \star \star}\end{array}$ \\
\hline
\end{tabular}

Note: ${ }^{\star} \mathrm{p}<.05,{ }^{* \star} \mathrm{p}<.01,{ }^{* \star *} \mathrm{p}<.001 ; \mathrm{X}_{\mathrm{MH}}{ }^{2}$

Among drinking behaviors considered acceptable by solitary drinkers, the highest reported percentage was for "drinking alone" (85.2\%), followed by "getting drunk" (42.6\%), "drinking in a convenience store" (38.1\%), and "drinking during the day" (37.0\%). Among social drinkers, the highest percentage was also reported for "drinking alone" (69.1\%), followed by "getting drunk" (37.9\%), "drinking in a convenience store" (35.2\%), and "drinking during the day" (31.5\%). However, non/light drinkers showed much less agreement with all statements than solitary and social drinkers: The highest percentages were reported for "drinking alone" (61.1\%), followed by "drinking during the day" (25.1\%), "getting drunk" (25.0\%), and "drinking in a convenience store" (24.6\%) (see Table 2).

\section{Drinking Motives Between Solitary And Social Drinkers}

Only respondents who consumed alcohol at least once or more per month responded to the questions concerning drinking motives. Drinking motives are important factors influencing choices, patterns, and outcomes regarding alcohol. The drinking motives of solitary and social drinkers are presented in Table 3. Solitary drinkers showed more agreement with all statements than did social drinkers. Among solitary drinkers, the main motive was "drinking helps to overcome work and life stress" (57.2\%), followed by "I drink to feel good/happy" (51.6\%), "alcohol makes food taste better," (39.3\%), and "I want to fall asleep quickly" (37.2\%). Among social drinkers, the highest number of participants agreed that "drinking helps to overcome work and life stress" (44.2\%), followed by "I drink to feel good and happy" (43.6\%), "alcohol makes food taste better" (28.0\%), and "I want to get rid of my fears and be brave" (15.7\%). In terms of social motives, there were no significant differences between solitary and social drinkers. Concerning environmental motives, solitary drinkers endorsed the following items more than social drinkers did: "many stores sell alcohol," $66.3 \%$ versus $62.5 \%$; "there are many places to drink," $31.3 \%$ versus $25.1 \%$; and "the price of alcohol is low," $17.5 \%$ versus $11.9 \%$ (see Table 3 ). 
Drinking motives by drinking type (\% of agreement) $(\mathrm{N}=2,426)$

\begin{tabular}{|c|c|c|c|c|}
\hline \multicolumn{2}{|l|}{ Motives } & \multirow{2}{*}{ 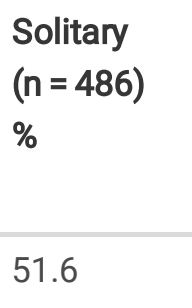 } & \multirow{2}{*}{$\begin{array}{l}\text { Social } \\
(n= \\
1,940) \\
\%\end{array}$} & \multirow{2}{*}{$\begin{array}{c}\mathrm{X}_{\mathrm{MH}}{ }^{2} \\
14.9^{\star * \star}\end{array}$} \\
\hline Personal & I drink to feel good/happy. & & & \\
\hline & I drink because there are few things to enjoy except drinking. & 25.7 & 16.9 & $26.0^{\star \star \star}$ \\
\hline & I drink because alcohol makes food taste better. & 39.3 & 28.0 & $31.9^{\star \star \star}$ \\
\hline & I drink to overcome work and life stress. & 57.2 & 44.2 & 3.2 \\
\hline & $\begin{array}{l}\text { When I cannot fall asleep easily, I drink because I want to fall } \\
\text { asleep quickly. }\end{array}$ & 37.2 & 15.7 & $134.7^{\star \star \star}$ \\
\hline & I drink when I want to get rid of my fears and be brave. & 25.3 & 19.7 & $11.9^{\star *}$ \\
\hline & Mean (SD) & $3.04(0.03)$ & $2.71(0.02)$ & $\begin{array}{l}\mathrm{F}= \\
3.83^{* \star \star}\end{array}$ \\
\hline \multirow[t]{4}{*}{ Social } & $\begin{array}{l}\text { I drink with others to become friends quickly and to get along with } \\
\text { them. }\end{array}$ & 57.2 & 57.3 & 2.1 \\
\hline & I drink to deal with difficult relationships. & 50.8 & 48.4 & 0.9 \\
\hline & I drink a lot because of work-related gatherings. & 21.0 & 23.6 & 3.2 \\
\hline & Mean (SD) & $3.15(0.03)$ & $3.12(0.02)$ & $F=0.35$ \\
\hline \multirow[t]{4}{*}{ Environmental } & $\begin{array}{l}\text { I drink to enjoy low-cost recreation because the price of alcohol is } \\
\text { low. }\end{array}$ & 17.5 & 11.9 & $18.8^{\star \star \star}$ \\
\hline & $\begin{array}{l}\text { I drink because there are many stores where I can buy alcoholic } \\
\text { beverages anytime. }\end{array}$ & 66.3 & 62.5 & 4.5 \\
\hline & I drink because there are many places to drink everywhere. & 31.3 & 25.1 & $8.8^{*}$ \\
\hline & Mean (SD) & $3.03(0.03)$ & $2.87(0.02)$ & $\begin{array}{l}F= \\
16.75^{\star \star \star}\end{array}$ \\
\hline $\begin{array}{l}\text { Note: }{ }^{*} p<.05,{ }^{*} \\
\text { at least once } p\end{array}$ & $\begin{array}{l}01,{ }^{* \star *} p<.001 \text {; Questions about drinking motives were only askec } \\
\text { onth. }\end{array}$ & articipan & o consu & alcohol \\
\hline
\end{tabular}

\section{Drinking frequency (number), alcohol consumption amount, and alcohol- related harm/troubles between solitary and social drinkers}

We assessed the drinking frequency (number) and alcohol amount for solitary and social drinkers. A MANCOVA, with gender as a covariate, was used to compare social and solitary drinkers in terms of frequency of drinking occasions per month and alcohol consumption per week. The means of drinking frequency for solitary and social drinkers were 6.1 and 3.6 per month, respectively $\left(F=131.94, p<.001, \eta^{2}=.098\right)$. The mean consumption of pure alcohol (ethanol) per week was $69.5 \mathrm{~g}$ and $46.8 \mathrm{~g}$, respectively $\left(F=160.52 p<.001, \eta^{2}=.117\right)$. Solitary drinkers drank more frequently than social drinkers, and they consumed significantly more alcohol. The pattern of solitary drinking behavior is likely to be predictive of high alcohol consumption (see Table 4). 
Table 4

Frequency of drinking occasions (number), alcohol consumption, and alcohol-related problems $(\mathrm{g})(\mathrm{N}=2,426)$

\begin{tabular}{|c|c|c|c|c|c|c|c|}
\hline & \multicolumn{2}{|c|}{$\begin{array}{l}\text { Solitary } \\
(n=486)\end{array}$} & \multicolumn{2}{|c|}{$\begin{array}{l}\text { Social } \\
(n=1,940)\end{array}$} & \multicolumn{2}{|c|}{$\begin{array}{l}\text { Total } \\
(\mathrm{N}=2,426)\end{array}$} & \multirow[b]{2}{*}{$F\left(\eta^{2}\right)$} \\
\hline & M & (SD) & M & $(\mathrm{SD})$ & M & $(\mathrm{SD})$ & \\
\hline $\begin{array}{l}\text { Frequency of drinking occasions per month } \\
\text { (number) }\end{array}$ & 6.14 & $(5.46)$ & 3.65 & $(3.92)$ & 4.15 & $(4.39)$ & $131.94^{\star \star \star}(0.098)$ \\
\hline \multirow[t]{2}{*}{ Amount of alcohol consumption per week (g) } & 69.54 & (89.39) & 46.82 & $(25.74)$ & 41.3 & (78.79) & $160.52^{\star \star \star}(0.117)$ \\
\hline & $\mathrm{n}$ & $\%$ & $\mathrm{n}$ & $\%$ & $\mathrm{~N}$ & $\%$ & $X_{M H^{2}}(p)$ \\
\hline $\begin{array}{l}\text { Legal trouble related to my drinking and } \\
\text { driving }\end{array}$ & 7 & 1.4 & 16 & 0.8 & 23 & 0.9 & $1.57(.210)$ \\
\hline Harmful effects on my work & 105 & 21.6 & 420 & 21.6 & 525 & 21.6 & $0.00(.983)$ \\
\hline Getting into fights while drinking & 59 & 12.1 & 197 & 10.2 & 256 & 10.6 & $1.62(.203)$ \\
\hline Destroying property & 12 & 2.5 & 25 & 1.3 & 37 & 1.5 & $3.61(.058)$ \\
\hline Injury during work or daily life & 14 & 2.9 & 55 & 2.8 & 69 & 2.8 & $0.00(.957)$ \\
\hline $\begin{array}{l}\text { Sexual harassment/unwanted sexual } \\
\text { behavior }\end{array}$ & 8 & 1.6 & 42 & 2.2 & 50 & 2.1 & $0.52(0.472)$ \\
\hline Blacking out & 138 & 28.4 & 505 & 26.0 & 643 & 26.5 & $1.12(.291)$ \\
\hline \multicolumn{8}{|c|}{$\begin{array}{l}\text { Note: The frequency of drinking occasions per month and degree of alcohol consumption per week were square-root- } \\
\text { transformed prior to the analyses. Means and standard deviations shown are for the untransformed variables. Univariate } \\
\text { test results reported are from Analyses of Covariance (ANCOVA). Gender was controlled for in all analyses. } \eta^{2}=\text { effect size } \\
\text { (eta squared); }{ }^{\star \star} p<.001\end{array}$} \\
\hline
\end{tabular}

There were no significant differences in alcohol-related harms or troubles between solitary and social drinkers. For both groups, the biggest problem was "blacking out" ( $28.4 \%$ for solitary vs. $26.0 \%$ for social), followed by "having trouble with work" (21.6\% for both), and "having a fight while drinking" (12.1 vs. 10.2\%).

\section{Factors associated with drinking frequency and alcohol amount between solitary and social drinkers}

A hierarchical regression analysis was conducted to determine factors associated with drinking frequency and alcohol amount of solitary vs. social drinkers (see Table 5). In the first step, socio-demographic variables (gender, marital status, education status, and household) and dependent variables (drinking frequency and alcohol consumption amount) were entered into the model. In the second step, four variables (drinking norms; personal, social, and environmental motives) were entered into the model. 
Table 5

Factors associated with drinking frequency and alcohol amount

Solitary Drinker $(n=486)$

\begin{tabular}{|c|c|c|c|c|c|c|c|c|c|c|c|c|}
\hline & \multicolumn{3}{|c|}{ Frequency } & \multicolumn{3}{|c|}{$\begin{array}{l}\text { Alcohol consumption } \\
\text { amount }\end{array}$} & \multicolumn{2}{|c|}{ Frequency } & \multicolumn{4}{|c|}{$\begin{array}{l}\text { Alcohol consumption } \\
\text { amount }\end{array}$} \\
\hline & $\mathrm{R}^{2}$ & B & $\begin{array}{l}\text { SE } \\
ß\end{array}$ & $\mathrm{R}^{2}$ & ß & $\begin{array}{l}\text { SE } \\
B\end{array}$ & $\mathrm{R}^{2}$ & ß & $\begin{array}{l}\text { SE } \\
ß\end{array}$ & $\mathrm{R}^{2}$ & B & $\begin{array}{l}\text { SE } \\
ß\end{array}$ \\
\hline \multicolumn{13}{|l|}{$\begin{array}{l}\text { Demographic } \\
\text { variables }\end{array}$} \\
\hline Gender & $.09^{\star \star \star}$ & $0.16^{\star \star \star}$ & 0.47 & $.10^{\star \star \star}$ & $0.16^{\star \star \star}$ & 0.10 & $.06^{\star \star \star}$ & $0.15^{\star \star \star}$ & 0.17 & $.08^{\star \star \star}$ & $0.16^{\star \star \star}$ & 0.10 \\
\hline Marital status & & 0.06 & 0.47 & & 0.06 & 0.10 & & 0.04 & 0.18 & & 0.02 & 0.10 \\
\hline $\begin{array}{l}\text { Education } \\
\text { status }\end{array}$ & & $0.10^{*}$ & 0.26 & & $0.11^{*}$ & 0.05 & & -0.04 & 0.09 & & $-0.04^{*}$ & 0.05 \\
\hline Job & & -0.02 & 0.59 & & -0.05 & 0.12 & & $-0.08^{\star \star \star}$ & 0.21 & & -0.08 & 0.12 \\
\hline $\begin{array}{l}\text { Household } \\
\text { income }\end{array}$ & & 0.05 & 0.27 & & 0.06 & 0.06 & & $0.04^{*}$ & 0.10 & & 0.05 & 0.06 \\
\hline $\begin{array}{l}\text { Attitude } \\
\text { variables }\end{array}$ & $.22^{\star \star \star}$ & & & $.26^{\star \star \star}$ & & & $0.19^{\star \star \star}$ & & & $0.24^{\star \star \star}$ & & \\
\hline $\begin{array}{l}\text { Drinking } \\
\text { norms }\end{array}$ & & 0.03 & 0.52 & & 0.04 & 0.11 & & $0.09^{\star \star \star}$ & 0.18 & & 0.09 & 0.11 \\
\hline $\begin{array}{l}\text { Personal } \\
\text { motives }\end{array}$ & & $0.42^{\star \star \star}$ & 0.48 & & $0.43^{\star \star \star}$ & 0.10 & & $0.30^{\star \star \star}$ & 0.17 & & $0.33^{\star \star \star}$ & 0.10 \\
\hline $\begin{array}{l}\text { Social } \\
\text { motives }\end{array}$ & & -0.09 & 0.42 & & -0.05 & 0.09 & & 0.02 & 0.15 & & 0.04 & 0.09 \\
\hline $\begin{array}{l}\text { Environmental } \\
\text { motives }\end{array}$ & & -0.02 & 0.38 & & -0.03 & 0.08 & & -0.01 & 0.14 & & -0.01 & 0.08 \\
\hline
\end{tabular}

Social Drinker $(n=1,940)$

Note: Gender (men = 1, women = 0), Marital status (married = 1, single/divorced $/$ widow $=0$ ), Job $($ No job $=1$, Had a job $=0$ )

Regarding drinking frequency, gender, education, and personal drinking motives were significant for solitary drinkers $\left(R^{2}=.22, p\right.$ $<.001$ ), while gender, job status, household income, drinking norms, and personal drinking motives were significant for social drinkers $\left(R^{2}=.19, p<.001\right)$. Regarding alcohol consumption amount, gender, education, and personal drinking motives were significant for solitary drinkers $\left(R^{2}=.26, p<.001\right)$, while gender, job status, household income, drinking norms, and personal drinking motives were significant for social drinkers $\left(R^{2}=.24, p<.001\right)$.

The variables of job status, household income, and drinking norms were significantly associated with drinking frequency only for social drinkers, not for solitary drinkers. Education was significantly associated with drinking frequency and consumption amount for solitary drinkers. For social drinkers, education was related to drinking consumption amount but not drinking frequency. The regression analysis revealed that personal motives for drinking were the most important factor of drinking frequency and consumption for both solitary and social drinkers, while other drinking motives were not (see Table 5).

\section{Discussion}

This study compared the social norms, motives, frequencies, and amounts of drinking among people who reported different drinking types. The major findings of the study are as follows.

When comparing solitary drinkers with social ones, it was found that socially vulnerable people are more likely to drink alone. Solitary drinkers are more likely to be divorced or separated, less educated, and marginally employed (people with temporary

Page 9/12 
jobs, rather than regular; the self-employed; and people with service- and sales-related jobs). Socially vulnerable people often resort to alcohol and experience alcohol-related harms owing to a lack of alternative resources when faced with hardship [18]. As a result, solitary drinkers may be more susceptible to alcohol-related problems than social drinkers.

There was a distinct difference in the type of drinking (solitary or social) in terms of social norms and motives for drinking. Solitary drinkers were more permissive toward drinking behaviors in response to all survey questions, including those regarding public drinking, daytime drinking, and drunkenness, than were social drinkers.

Regarding their motives for drinking, it was found that solitary drinkers had a higher level of drinking motivation, both to enhance positive emotions and to cope with negative ones, than social drinkers. However, there are some discrepancies in previous studies: Several studies have shown that solitary drinkers have higher levels of coping with negative affects and lower levels of enhancing positive affects [11-13], while others show that solitary drinkers are higher in all positive or negative expectancies [12-13]. This finding supports that solitary drinkers are less confident of their drinking control in positive and negative situations. There were no significant differences between the two groups in terms of social drinking motives. This finding is consistent with results of other studies showing that solitary drinkers drink alone to cope with their emotions, not because they have fewer social interaction motives, or are in a situation in which they have no choice but to drink alone[11][15]. Lastly, solitary drinkers had higher levels of environmental drinking motives stimulated by their surroundings. This finding shows that solitary drinkers are more tempted by their surroundings to buy and drink alcohol than social drinkers. The concentration of stores and bars in South Korea therefore constitutes a threat to public health that is amenable to change through alcohol policy reform.

The results of this study indicate that solitary drinkers tend to consume more alcohol and to consume it more frequently than social drinkers, drinking 6.1 times a month, on average, vs. 3.6 times per month for social drinkers. More frequent drinking by solitary drinkers leads to a higher amount of alcohol consumption: about 69.54 grams a week compared to 46.82 grams for social drinkers. These findings provide support for the hypothesis that drinking frequency and alcohol consumption amount are associated with drinking patterns.

In terms of alcohol-related harms and troubles, there are no differences between solitary and social drinkers. Some studies also pointed to the effect of the context in which drinking takes place on the occurrence of alcohol-related problems. Solitary drinking is a specific drinking context, characterized by the lack of a companion to provide some social control when drinking. However, there have been few studies of different drinking patterns and drinking contexts, so very little is known about solitary drinking and the alcohol-related harms and troubles associated with it [19].

Solitary drinking is a public health issue that should be afforded more attention, as solitary drinkers tend to drink regularly, which may lead to alcohol use disorder, while social drinkers tend not to do so on a regular basis [8]. According to previous studies, solitary drinkers experience stress and depression more often than social drinkers, a problem that should be given more attention $[11,15,20]$. Accordingly, an intervention to help solitary drinkers cope with negative life events or stressful situations in a sensible way and to provide them with social supports is needed [15].

Despite meaningful findings on solitary drinkers in South Korea, this study has some limitations in terms of the measures of solitary drinking and alcohol-related harms. The self-reported measure may have underestimated both solitary drinking and alcohol-related harms. We asked participants to categorize themselves as solitary drinkers or social drinkers, but this may not be a good measure because solitary drinking can be under-reported owing to generalized social disapproval. More research is therefore needed to develop valid measures of solitary drinking behavior. Furthermore, we did not use a standardized alcoholrelated harms tool for South Korea, but it is necessary to develop one.

\section{Conclusion}

By identifying the differences between social drinking and solitary drinking, which has become increasingly widespread in recent years, this study will be helpful in establishing future strategies to prevent drinking problems. Contrary to the findings of 
the study conducted by the Korean Food and Drug Administration in 2016 [16] showing that solitary drinkers drink a smaller quantity of alcohol than social drinkers when they drink, this study shows that solitary drinkers drink more in terms of both frequency and consumption, suggesting that more intensive attention should be given to this group. Drinking to relieve depression and other negative emotions cannot be a long-term solution, despite the temporary relief it offers; on the contrary, in the long term, it can worsen feelings of depression and negative emotions, which can lead to a vicious cycle of continued excess. In particular, solitary drinking can induce more frequent and more regular drinking, which can eventually lead to alcohol dependence. It is evident that appropriate interventions for solitary drinkers who drink to deal with emotional problems are urgently needed.

\section{Declarations}

Authors' contributions

All authors made substantial contributions to analysis and interpretation of the data. All authors participated in drafting the manuscript and revising it critically for important intellectual content. AS was in charge of revising the manuscript and giving final approval of the version to be published.

Funding

This research funded by the Incheon National University Research Grant in 2018.

Availability of data materials

Data will be made available upon request.

Ethics approval and consent to participate

Our survey instrument followed the guidelines of the institutional Review Board of Sahmyook University (2-7001793-AB-N012018015HR), registered on April 17, 2018). All procedures were performed in accordance with the ethical standards of Declaration of Helsinki, Informed consent was obtained from all individual participants included in the survey in written form.

Consent for publication

All authors give consent for publication.

Competing interest

The authors declare no conflicts of interest.

Author Details

${ }^{1}$ Dept. of, Incheon University, Incheon City, South Korea

Page $11 / 12$ 
${ }^{2}$ Dept. of Health \& Human Performance, Sahmyook University, Seoul, Korea

\section{References}

1. WHO. Global status report on alcohol and health. WHO; 2018.

2. OECD. Health at a Glance 2017: OECD publishing; 2017.

3. OECD. Tackling Harmful Alcohol Use: OECD publishing; 2015.

4. Kwang Kee K, Jung J, Ji Hyun L. Drinking behaviors and policies to reduce harms caused by alcohol use and health promotion policy. Korean J Health Educ Promo. 2016;33(4):21-34.

5. KCDC. Korea health statistics 2017. 2017.

6. Franco S. Tackling harmful alcohol use economics and public health policy: Economics and public health policy: OECD publishing; 2015.

7. Sohn A. A study of the characteristics of drinking culture and the policy for the improvement of drinking availability. Sejong: Ministry of Health \& Welfare (MW), 2018.

8. Lee H, Seok B, Sohn A. The role of social media content on solitary drinking among Korean adults. Cyberpsychol Behav Soc Netw. 2019;22(6):397-403.

9. Ko S, Sohn A. Behaviors and culture of drinking among Korean people. Iran J Public Health. 2018;47(Suppl 1):47.

10. Cooper ML. Motivations for alcohol use among adolescents: Development and validation of a four-factor model. Psychol Assess. 1994;6(2):117-28.

11. Gonzalez VM, Collins RL, Bradizza CM. Solitary and social heavy drinking, suicidal ideation, and drinking motives in underage college drinkers. Addict Behav. 2009;34(12):993-9.

12. Christiansen M, Vik PW, Jarchow A. College student heavy drinking in social contexts versus alone. Addict Behav. 2002;27(3):393-404.

13. Tucker JS, Ellickson PL, Collins RL, Klein DJ. Does solitary substance use increase adolescents' risk for poor psychosocial and behavioral outcomes? A 9-year longitudinal study comparing solitary and social users. Psychol Addict Behav. 2006;20(4):363.

14. Jin K, Sohn A, Kim S, Kim Y, Hong Y. Gender difference in drinking norms and motives in South Korea. Korean J Health Educ Promo. 2018;35(1):13-24.

15. Lee H. Solitary drinking vs. social drinking: Analysis of mental health in heavy drinkers Alcohol \& Health Behav Res. 2017;18(2):1-14.

16. KFDA. In the age of solitary drinker, the increase of solitary drinking. Osong: Korean Food and Drug Administration, 2016. Contract No.: 26 Feb.

17. Sohn A. Development of an assessment tool for drinking norms and motives among Korean adults. Alcohol \& Health Behav Res. 2019;20(1):1-11.

18. Cho B, Sohn A, Kim M, Yang J, Son S. Determinants of drinking and harmful drinking experience among Korean adults. Alcohol \& Health Behav Res. 2018;19(2):1-14.

19. Creswell KG, Chung T, Wright A, Clark DB, Black JJ, Martin CS. Personality, negative affect coping, and drinking alone: a structural equation modeling approach to examine correlates of adolescent solitary drinking. Addiction. 2015;110(5):775783.

20. Bourgault C, Demers A. Solitary drinking: a risk factor for alcohol-related problems? Addiction. 1997;92(3):303-12.

21. Cooper ML, Frone MR, Russell M, Mudar P. Drinking to regulate positive and negative emotions: a motivational model of alcohol use. J Pers Soc Psychol.1995;69(5):990-1005. 